\title{
28 Research Square \\ Frontline Physician Burnout During the COVID-19 Pandemic: National Survey Findings
}

Joy Melnikow ( $\square$ jamelnikow@ucdavis.edu )

University of California, Davis

Andrew Padovani

University of California, Davis

Marykate Miller

University of California, Davis

\section{Research Article}

Keywords: COVID-19, frontline physicians, physician burnout, physician wellbeing

Posted Date: October 25th, 2021

DOI: https://doi.org/10.21203/rs.3.rs-966072/v1

License: (c) (1) This work is licensed under a Creative Commons Attribution 4.0 International License.

Read Full License

Version of Record: A version of this preprint was published at BMC Health Services Research on March 19th, 2022. See the published version at https://doi.org/10.1186/s12913-022-07728-6. 


\section{Abstract}

Background: Physician burnout and wellbeing are an ongoing concern. Limited research has reported on the impact of the COVID 19 pandemic on burnout among U.S. physicians.

Methods: We surveyed U.S. frontline physicians at two time points (wave one in May-June 2020 and wave two in Dec 2020-Jan 2021) using a validated burnout measure. The survey was emailed to a national stratified random sample of family physicians, internists, hospitalists, intensivists, emergency medicine physicians, and infectious disease physicians. Burnout was assessed with the Professional Fulfillment Index Burnout Composite scale (PFI-BC). Quantile regression was used to estimate the change in median PFI-BC scales, adjusting for physician, geographic, and pandemic covariates.

Results: Overall, $38.1 \%$ of 286 respondents reported PFI-BC scores consistent with burnout in wave one, and $33.1 \%$ of 262 respondents in wave two. Burnout declined or was unchanged among all specialties surveyed apart from hospitalists. $34.2 \%$ of primary care physicians reported burnout in wave one and $29.7 \%$ in wave two. Burnout declined among critical care (53.5\% to $43.1 \%)$ and emergency physicians ( $50.6 \%$ to $44.5 \%$ ); but hospitalist burnout rates increased ( $27 \%$ to $44.2 \%$ ).

Conclusions: Rates of physician burnout during the first year of the pandemic appear to be comparable to pre-pandemic rates. We found no evidence of increasing burnout over a six month period, apart from hospitalist respondents. Impacts of the ongoing pandemic on physician burnout warrant continued research.

\section{Background}

Physician burnout has received growing attention in recent years. ${ }^{1}$ The COVID-19 pandemic presents an unprecedented healthcare challenge to the world, and international research has indicated that healthcare workers have experienced depression, anxiety, post-traumatic stress symptoms and greater burnout. ${ }^{2-7}$ More limited research has reported on the pandemic's impact on U.S. physician burnout; most surveys have focused on single health systems or single specialties. ${ }^{8-10}$ One national survey solicited survey respondents via social media to evaluate the incidence of depressive symptoms, anxiety, and PTSD at a single time point. ${ }^{11}$ As the pandemic has continued, there is a need to track the impact on physician burnout over time. We conducted an online survey at two time points during the pandemic using a validated measure of burnout ${ }^{12}$ in a national stratified sample of U.S. physicians practicing in frontline specialties.

\section{Methods}

A stratified random sample of 10,000 US physicians from a comprehensive list (AMA Physician Masterfile) included 4,000 primary care physicians (2,000 family physicians and 2,000 internists), 1,000 hospitalists, 2,000 critical care and pulmonary intensivists, 2,000 emergency medicine physicians, and 
1,000 infectious disease physicians. Hospitalists, intensivists, infectious disease and emergency medicine physicians were proportionally oversampled to ensure that responses represented these frontline specialties. To adjust for sampling and nonresponse biases, survey weights were constructed as the product of scaled sampling weights and scaled nonresponse weights. ${ }^{13}$ We assessed burnout over the previous two weeks with the validated Professional Fulfillment Index Burnout Composite scale (PFI$B C)^{12}$ via an emailed Qualtrics ${ }^{\text {TM }}$ survey sent in May-June 2020 and January-February 2021. Responses were analyzed with Stata/MP $17^{\mathrm{TM}}$.

The PFI-BC scale averages the work exhaustion and interpersonal disengagement scales. Items are rated from "Not at all" (0) to "Extremely" (4). Respondents were defined as experiencing burnout if their average score exceeded 1.4, previously defined as a threshold for burnout. ${ }^{12}$ We estimated changes for median PFI-BC scores by quantile regression of PFI-BC scores on a set of physician, geographic, and pandemic covariates using weighted data. $95 \%$ confidence intervals for the average predicted changes were estimated via bootstrapping. ${ }^{14}$

Open response questions inquired about how physicians' lives had changed with the pandemic, and what would make things better for their patients and in their work.

\section{Results}

286 responses were collected in May-June 2020 (wave one) and 262 in January-February 2021 (wave two). Characteristics of respondents and nonrespondents are compared in Table 1. The median weighted PFI-BC score was unchanged at 1.0 (95\% Cl 0.8-1.2) across both waves. In weighted results, $38.1 \%$ of 286 respondents reported PFI-BC scores of at least 1.4 (the threshold indicating burnout) in wave one, and $33.1 \%$ of 262 respondents scored at least 1.4 in wave two. In wave one, $53.5 \%$ of critical care and $50.6 \%$ of emergency physicians scored at least 1.4; in winter, wave two rates of burnout declined to $43.1 \%$ and $44.5 \%$, respectively. Infectious disease physicians reported above average burnout in both waves of the survey with $42.7 \%$ scoring in the burnout range in wave one and $37.2 \%$ in wave two. Primary care physicians reported relatively less burnout compared to other specialties with limited change over time: $34.2 \%$ scored above the threshold in wave one and $29.7 \%$ in wave two. Burnout among hospitalists, however, increased from $27 \%$ in wave one to $44.2 \%$ in wave two.

Figure 1 shows the average predicted change in median PFI-BC scores between wave 1 (May-June 2020) and wave 2 (December 2020 - January 2021) for each specialty, with $95 \%$ confidence intervals. $45.2 \%$ of women reported scores consistent with burnout compared to $33.6 \%$ of men in wave one, but 6 months later in wave two, $31.1 \%$ of women scored above the burnout threshold compared to $34.2 \%$ of men.

In open-ended comments from the first wave survey, the isolation imposed by the pandemic was a prominent theme. Many respondents commented on the visitor restriction impacts for very ill patients: "The most difficult part of working in the ICU was having patients struggle and die without family members to hold their hands, that was very sad." 
Some physicians isolated themselves from their own families to protect them: "While living in the same house I have a separate room and entry/exit door, if I wasn't in the hospital I was in my room. Eat dinner sitting outside the dining room window in my yard while my wife and kids sat on the other side of the window at the table, so we can have a family meal. Facetime my kids to help them with homework."

When asked what changes or modifications would most help them in their work, first wave respondents described a need for more PPE, access to rapid testing for patients, and clear national prevention and treatment guidelines. In the second wave, respondents emphasized increased vaccine availability, clear public health messaging on vaccine effectiveness, and infection prevention measures. In both waves, some respondents across specialties emphasized the importance of the public wearing masks to prevent transmission.

\section{Discussion}

We hypothesized high rates of burnout would worsen over time among frontline physicians during the pandemic, but this was not confirmed by our findings. The overall rate of burnout did not increase in wave 2 responses relative to wave 1, possibly due to institutional or personal adaptations to the pandemic and the beginning of vaccine rollout in winter 2021. As the pandemic continues in spite of available vaccines, frontline physician burnout may yet worsen.

In 2019 (before the pandemic), Brady et al. surveyed physicians across all specialties in an AMA Masterfile sample and reported that $44.5 \%$ (599) scored 1.4 or greater on the PFI-BC; ${ }^{15}$ in the current study, $40 \%$ (184) of the frontline physicians we surveyed during the pandemic scored $\geq 1.4$ on the burnout scale in at least one survey wave, suggesting that the relatively high rate of physician burnout prepandemic may not have increased.

Our findings are limited by low response rates and potential selection bias; physicians most subject to burnout may be less likely to respond a survey. Studies from around the world have emphasized the mental health impacts of the pandemic on healthcare worker anxiety, depression, and PTSD. ${ }^{2}$ However, the need for a crisis response by physicians may also have been protective. Hartzband and Groopman note the pandemic led to an "astounding display of selflessness by healthcare professionals" and restoration of autonomy, competency, and relatedness in a national health crisis may have countered physician burnout trends. ${ }^{16}$

Our finding that burnout rates declined for emergency medicine and critical care physicians, while increasing for hospitalists deserves additional research. With the limited uptake of the COVID-19 vaccines and the rapid spread of the Delta variant, all regions of the U.S. are have again faced increasing rates of infection. ${ }^{17}$ Hospitalists care for the greatest volume of inpatients with COVID-19, many of whom have long hospital stays. Higher initial rates of burnout in women physicians, who faced additional burdens at home and at work, ${ }^{18}$ also deserve investigation, as do personal and system adaptations that may have attenuated physician burnout during the pandemic. 


\section{Conclusions}

Physician burnout rates were a matter of great concern before the onset of the COVID-19 pandemic. We found limited changes in burnout through the pandemic's first year. Responding to a severe public health crisis may have been protective in some respects, however, as time passes and the pandemic continues, fatigue and frustration among frontline physicians may lead to worsening burnout.

\section{Declarations}

\section{Ethics approval and consent to participate}

This study was reviewed by the University of California Davis Institutional Review Board and determined to have methods in accordance with guidelines and regulations qualifying it as being a low risk study. As such, informed consent was obtained through the recipients' review of the email cover letter containing the link to the physician survey.

\section{Consent for publication}

Not applicable

\section{Availability of data and materials}

Fully deidentified datasets used and/or analyzed during the current study are available from the corresponding author on reasonable request.

\section{Competing interests}

The authors declare that they have no competing interests.

\section{Funding}

The study was funded by internal funds from the Center for Healthcare Policy and Research at the University of California, Davis.

\section{Authors' contributions}

JM conceived of the project, oversaw the survey design and data collection, interpretation of the analysis, and drafted the manuscript. AP designed and conducted the data analysis, participated in the interpretation of the results, and assisted with drafting the manuscript. MM contributed to the study design, collected the data, constructed the data base, and edited the manuscript. All authors reviewed the final version of the manuscript.

\section{ACKNOWLEDGEMENTS}


The authors thank the physicians who contributed their valuable time to respond to our surveys during an unprecedented pandemic.

\section{References}

1. Rotenstein LS, Torre M, Ramos MA, et al. Prevalence of burnout among physicians: a systematic review. JAMA. 2018;320(11):1131-1150.

2. Bekele F, Hajure M. Magnitude and determinants of the psychological impact of COVID-19 among health care workers: a systematic review. SAGE Open Med. 2021;9.

3. Dobson $\mathrm{H}$, Malpas $\mathrm{CB}$, Burrell AJ, et al. Burnout and psychological distress amongst Australian healthcare workers during the COVID-19 pandemic. Australas Psychiatry. 2021;29(1):26-30.

4. Lu MY, Ahorsu DK, Kukreti S, et al. The prevalence of post-traumatic stress disorder symptoms, sleep problems, and psychological distress among COVID-19 frontline healthcare workers in Taiwan. Front Psychiatry. 2021;12:705657.

5. Han S, Choi S, Cho SH, Lee J, Yun J-Y. Associations between the working experiences at frontline of COVID-19 pandemic and mental health of Korean public health doctors. BMC Psychiatry. 2021;21(1):298.

6. Bongelli R, Canestrari C, Fermani A, et al. Associations between personality traits, intolerance of uncertainty, coping strategies, and stress in Italian frontline and non-frontline HCWs during the COVID-19 pandemic - a multi-group path-analysis. Healthcare. 2021;9(8):1086.

7. Li WQ, Yuan P, Sun J, et al. Resilience, coping style, and COVID-19 stress: effects on the quality of life in frontline health care workers. Psychol Health Med. 2021:1-13.

8. Kachadourian LK, Feder A, Murrough JW, et al. Transdiagnostic psychiatric symptoms, burnout, and functioning in frontline health care workers responding to the COVID-19 pandemic: a symptomics analysis. J Clin Psychiatry. 2021;82(3).

9. Gray BM, Vandergrift JL, Barnhart BJ, et al. Changes in stress and workplace shortages reported by U.S. critical care physicians treating coronavirus disease 2019 patients. Crit Care Med. 2021;49(7):1068-1082.

10. Baumann BM, Cooper RJ, Medak AJ, et al. Emergency physician stressors, concerns, and behavioral changes during COVID-19: a longitudinal study. Acad Emerg Med. 2021;28(3):314-324.

11. Young KP, Kolcz DL, O'Sullivan DM, Ferrand J, Fried J, Robinson K. Health care workers' mental health and quality of life during COVID-19: results from a mid-pandemic, national survey. Psychiatr Serv. 2021;72(2):122-128.

12. Trockel $M, B o h m a n ~ B$, Lesure $E$, et al. A brief instrument to assess both burnout and professional fulfillment in physicians: reliability and validity, including correlation with self-reported medical errors, in a sample of resident and practicing physicians. Acad Psychiatry. 2018;42(1):11-24.

13. Valliant R, Dever J, Kreuter F. Practical tools for designing and weighting survey samples. New York: Springer; 2013. 
14. Graubard BI, Korn EL. Predictive margins with survey data. Biometrics. 1999;55(2):652-659.

15. Brady KJS, Ni P, Carlasare L, et al. Establishing crosswalks between common measures of burnout in US physicians. J Gen Intern Med. 2021.

16. Hartzband P, Groopman J. Physician burnout, interrupted. N Engl J Med. 2020;382(26):2485-2487.

17. Coronavirus in the U.S.: latest map and case count. New York Times. August 24, 2021. https://www.nytimes.com/interactive/2021/us/covid-cases.html. Published August 24, 2021. Accessed August 24, 2021.

18. Jones Y, Durand V, Morton K, et al. Collateral damage: how COVID-19 is adversely impacting women physicians. J Hosp Med. 2020;15(8):507-509.

\section{Table}

Table 1. Comparison of sample characteristics by response status $(n=10,002)$

\begin{tabular}{|lllllll|}
\hline & \multicolumn{2}{l}{ Did not respond } & \multicolumn{2}{l}{ Responded } & \multicolumn{2}{l|}{ Total } \\
\hline & Count & Share & Count & Share & Count & Share \\
\hline Sex $(\mathrm{N}=10,0002)$ & & & & & & \\
\hline Male $(\mathrm{N}=6,598)$ & 6,340 & $66.4 \%$ & 258 & $57.2 \%$ & 6,598 & $66.0 \%$ \\
\hline Female $(\mathrm{N}=3,404)$ & 3,211 & $33.6 \%$ & 193 & $42.8 \%$ & 3,404 & $34.0 \%$ \\
\hline Specialty $(\mathrm{N}=10,0002)$ & & & & & & \\
\hline Emergency medicine $(\mathrm{N}=2,001)$ & 1,909 & $20.0 \%$ & 92 & $20.4 \%$ & 2,001 & $20.0 \%$ \\
\hline Critical care $(\mathrm{N}=2,000)$ & 1,899 & $19.9 \%$ & 101 & $22.4 \%$ & 2,000 & $20.0 \%$ \\
\hline Primary care $(\mathrm{N}=4,001)$ & 3,853 & $40.3 \%$ & 148 & $32.8 \%$ & 4,001 & $40.0 \%$ \\
\hline Hospitalist $(\mathrm{N}=1,000)$ & 946 & $9.9 \%$ & 54 & $12.0 \%$ & 1,000 & $10.0 \%$ \\
\hline Infectious disease $(\mathrm{N}=1,000)$ & 944 & $9.9 \%$ & 56 & $12.4 \%$ & 1,000 & $10.0 \%$ \\
\hline & Mean & Std. Dev. & Mean & Std. Dev. & Mean & Std. Dev. \\
\hline Years in practice $(\mathrm{N}=10,001)$ & 17.5 & 11.1 & 17.5 & 10.8 & 17.5 & 11.1 \\
\hline
\end{tabular}

\section{Figures}




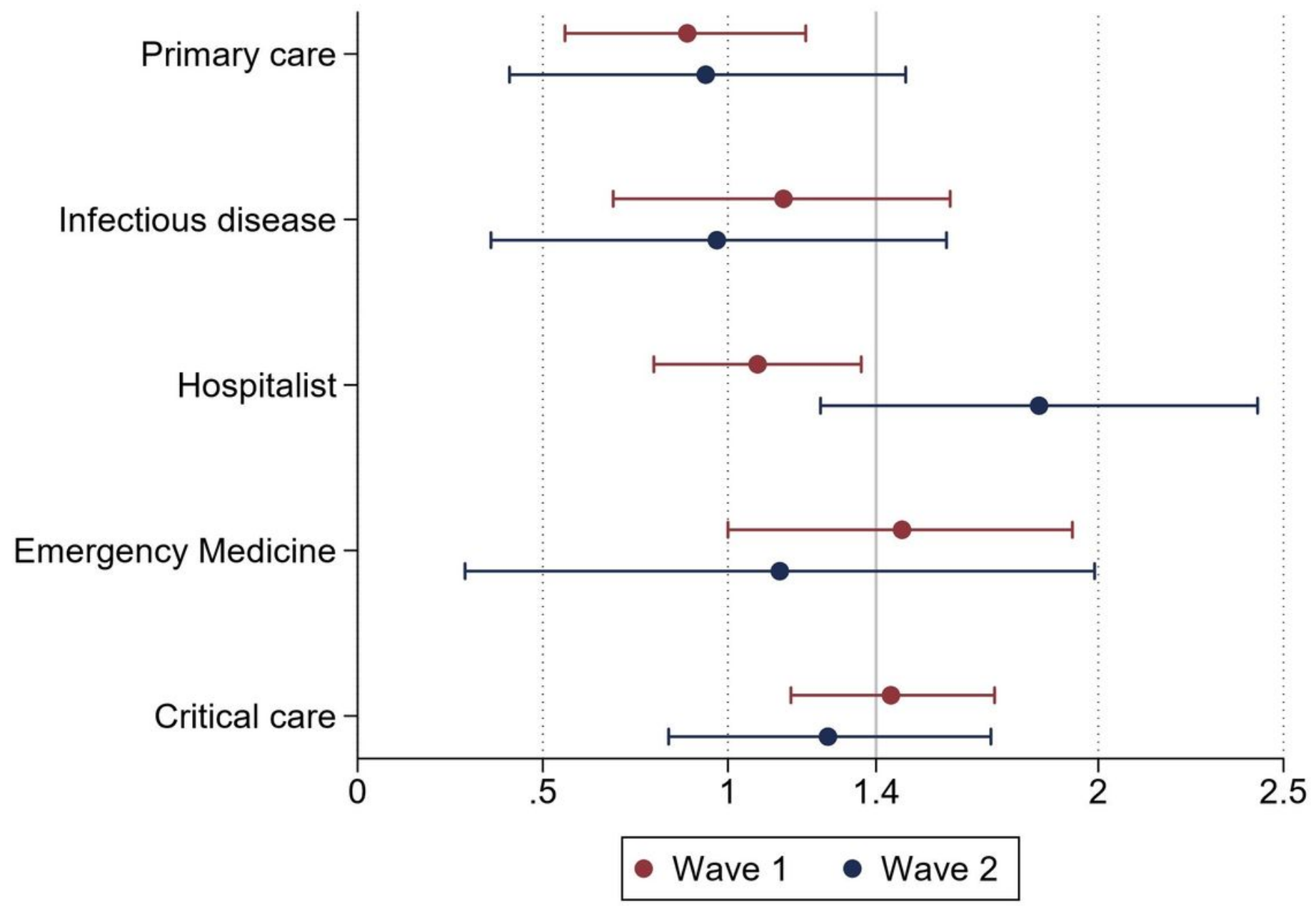

Reports regression-adjusted medians for each specialty, adjusted for physician, pandemic, and geographic covariates by quantile regression. Capped lines depict bootstrapped $95 \%$ confidence intervals.

\section{Figure 1}

Average predicted change in median Professional Fulfillment Index Burnout Composite (PFI-BC) Scores for each specialty surveyed. Scores greater than or equal to 1.4 are considered to indicate burnout. 